\title{
Doxorubicin-induced transcriptome meets interactome: identification of new drug targets
}

\author{
Hilal TAYMAZ-NIKKEREL* (D) \\ Department of Genetics and Bioengineering, Faculty of Engineering and Natural Sciences, İstanbul Bilgi University, İstanbul, Turkey
}

Received: 14.07.2021 • Accepted/Published Online: 20.12.2021 • Final Version: 05.04.2022

\begin{abstract}
The working mechanism of the chemotherapeutic drug doxorubicin, which is frequently used in cancer treatment, its effects on cell metabolism, and pathways activated solely by doxorubicin are not fully known. Understanding these principles is important both in improving existing therapies and in finding new drug targets. Here, I describe a systems-biology approach to find a generalizable working principle for doxorubicin by superimposition of human interactome over gene datasets commonly expressed among various cancer types. The common -in at least two different diseases-transcriptional response of distinctive cancer cell lines to doxorubicin was reflected via 199 significantly and differentially expressed genes, mostly related to the regulation of transcription. Then, by integrating with interactome data, an active network was constructed allowing detection of clusters. Since each cluster defines densely connected regions, another level of understanding of functional principles is provided. Significant clusters were associated with the linked transcription factors and transcriptional factor enrichment analysis within these regulatory networks led to the proposition of Pou5f1b, Znf428, Prmt3, Znf12, Erg, Tfdp1, Foxm1, and Cenpa as new drug targets in drug development that can be applied in different cancer types.
\end{abstract}

Key words: Bioinformatics, doxorubicin, interactome, systems biology, transcriptome

\section{Introduction}

One of the most used chemotherapeutic drugs, shown to be efficient in different types of cancer, is doxorubicin. Doxorubicin is known to intercalate into DNA, inhibit topoisomerase II, lead to DNA double-strand breaks, induce production of reactive oxygen species (ROS), overproduce ceramide, damage chromatin through histone modifications and disrupt nucleosome assembly (Yang et al., 2014; van der Zanden et al. 2020). Although it has a high drug efficacy, usage of doxorubicin creates resistance and causes several side effects (Ferreira et al., 2017; Ashrafizaveh et al., 2021). Despite the known functions of doxorubicin, the mechanism of action is not fully clarified. To overcome the limitations of the drug, to design better therapies and to utilize it in combination therapies, comprehensive understanding of the mechanism is required.

With the broad usage of transcriptome profiling, there have been studies suggesting signature genes that can be used to predict type of a disease, subtype and eventually the drug that can be administered for therapy, centering at cancer- and/or cell type-specific responses (Yu et al., 2019; Gopi and Kidder, 2021) or effects of various concentrations

\footnotetext{
*Correspondence: hilal.nikerel@bilgi.edu.tr
}

of the drug on a certain cell model (Maillet et al., 2016). Functional annotation and/or correlation analysis together with clustering methods on gene expression data are used to identify differences between tumors or cell lines. Focusing on the differences in gene expression, therefore, serves to improve the prediction of that certain type of disease's response. However, such approaches yield ad hoc results specific to the used data and/or platform used for the measurement of data leading to lack of generalization.

Integrative multiomic approaches were applied to understand doxorubicin-induced cardiotoxicity, to identify the common signature of anthracycline-induced cardiotoxicity, or to suggest patient-specific optimal combination therapy (Selevsek et al., 2020; Cava et al., 2021). Systems-wide effects of doxorubicin have been investigated also in yeast cells. While long-term treatment showed extensive reconfiguration of metabolic and signaling networks with ROS formation and DNA damage (Taymaz-Nikerel et al., 2018), short-term treatment showed the contribution of DNA repair, DNA replication, and RNA surveillance pathway (Karabekmez et al., 2021). Addition to the transcriptome and fluxome, interactome was integrated in those studies, which explained well the effects in eukaryotic yeast cells. Mentioned metaanalysis 
studies focused on the effects of doxorubicin in a certain cell type or differences across the set of various cells. However, a genome-wide approach to explore the common response of doxorubicin in different cancer types has not been applied.

Among the cancer types in which doxorubicin treatment has been applied either in single form or in combination with other chemotherapeutic drugs, are breast cancer (Di Francesco et al., 2021), leukemia (Turk et al., 2020), and nonsmall cell lung cancer (Shahriari et al., 2021). Though, doxorubicin is not much preferred in the treatment of colon cancer (Sonowal et al., 2017) or renal cancer (Acharya and Singh, 2021) due to severe cardiotoxiciy and drug resistance. These different sets of diseases are selected to examine the common effect(s) of doxorubicin regardless of the cancer/cell type. By this, affected processes, drug-specific transcriptional and regulatory networks in doxorubicin treatment irrespectively can be identified, and consensus mechanism of action can be offered.

Here, a systems biology approach is described, which is based on the integration of transcriptome with interactome data for the selected cell types, providing the regulatory components having a role in the mechanism of action of doxorubicin. Enrichment analyses relative to processes, pathways, and transcription factors demonstrate that there are several common types of machinery responsible for the cellular changes caused by doxorubicin. Moreover, such an approach enables the prediction of new targets for the development of drugs or of combination therapies.

\section{Materials and methods}

\subsection{Compilation of gene expression data}

While selecting gene expression datasets, cancer types, cell lines, dosage, and duration of administration of doxorubicin drug were taken into consideration. Microarray data, obtained using the Affymetrix platform, associated with doxorubicin was collected by scanning the NCBI Gene Expression Omnibus, GEO, and ArrayExpress, the functional genomics data repository supporting MIAME-compliant data submission.

Doxorubicin-induced transcriptome responses were then collected from the measurements across the National Cancer Institute (NCI)-60 cell line panel (Monks et al., 2018). In that study, expression of genes in NCI-60 human tumor cell lines were measured in response to several anticancer agents for 2, 6, and $24 \mathrm{~h}$. For renal cancer, breast cancer, leukemia, nonsmall cell lung cancer and colon cancer, the gene expression data, Gene Expression Omnibus (GEO) accession number of GSE116441 measured in the cells treated with $1000 \mathrm{~nm}$ of doxorubicin were used. As reference gene expressions, data measured in untreated control cultures were applied. Details of the compiled data are given in Supplementary Table S1.

\subsection{Data analysis}

2.2.1. Differential expression analysis in transcriptome First, datasets were normalized. Differentially expressed genes (DEGs) were defined from normalized logexpression values using linear models for microarray data. In data analysis, t-test method was applied to determine statistically significant (fold change in gene expression $>|1.05|$, p-value $<0.05$ ) subsets. These analyses were performed in CLC Genomics Workbench (Qiagen Bioinformatics).

\subsubsection{Integration with interactome data}

A protein-protein interaction (PPI) network was created using the proteins encoded by the common - in at least two diseases - differentially expressed genes and physical interactions of these proteins obtained from BIOGRID Homo sapiens 3.5.187 database comprising 613869 protein-protein interactions (Oughtred et al., 2019). Subnetwork analysis was carried out to detect clusters within the active network via MCODE application in Cytoscape 3.8.0, where all networks were visualized (Shannon et al., 2003).

\subsubsection{Functional annotation analysis}

Functional annotation analyses were carried out for the common differentially expressed genes and for each cluster identified within the active PPI network. Gene ontology (GO) term enrichment analyses were performed in DAVID functional annotation tool to identify significantly (Benjamini-Hochberg corrected p-value < $0.05)$ associated biological process, molecular function, cellular compartment, and KEGG pathways (Huang et al., 2009).

\subsubsection{Transcription factor enrichment analysis}

For each cluster identified within the active PPI network, transcription factor (TF) enrichment analysis was done in ChEA3, a web-based tool, ranking TFs (Keenan et al., 2019).

\section{Results and discussion}

This study aims to explain the working principle of doxorubicin and, via this, propose new drug targets for cancer therapy. A systems biology approach was applied through a metaanalysis of available transcriptome data measured in the presence of doxorubicin subjected to different cell lines of cancer. Commonly altered genes were coupled with interactome yielding a mechanistic and systems-level understanding.

\subsection{Common genes differentially expressed under doxo-} rubicin treatment in different cancer types

Genome-wide response of renal cancer, breast cancer, leukemia, nonsmall cell lung cancer, and colon cancer cells to doxorubicin at the transcriptional level revealed that numerous genes were differentially and significantly (fold change $>|1.05|$ and $p$-value $<0.05)$ expressed. Number of 
identified differentially expressed genes (DEGs) for each cancer type are presented in Figure 1.

In order to determine the common DEGs of different cancer types, genes that showed significant differences in at least two diseases were identified. This resulted in 199 genes, listed in Supplementary Table S2. Gene ontology (GO)-term enrichment analyzes for these 199 common DEGs revealed statistically significant (BenjaminiHochberg $<0.05$ ) biological processes (Figure 2A), molecular functions (Figure 2B), and the cellular part they take place in (Figure 2C). TNF signaling pathway and osteoclast differentiation are significant pathways. When these findings are examined, it is seen that these common genes are mostly related to the regulation of transcription (Figure 2A).

\subsection{Construction of the active network}

An active network was formed by obtaining the proteinprotein interactions of the proteins encoded by the common 199 DEGs (Supplementary Table S2). After removing the unlinked residues, a linked network of active protein-protein interactions was identified through 7022 proteins and 15775 protein-protein interactions. Topological analysis of the active protein-protein interaction network revealed that it is a scale-free network,

\begin{tabular}{|l|c|}
\hline Cancer type & Number of DEGs \\
\hline Renal cancer & 314 \\
Breast cancer & 139 \\
Leukemia & 288 \\
Non-small cell lung cancer & 367 \\
Colon cancer & 459 \\
\hline
\end{tabular}

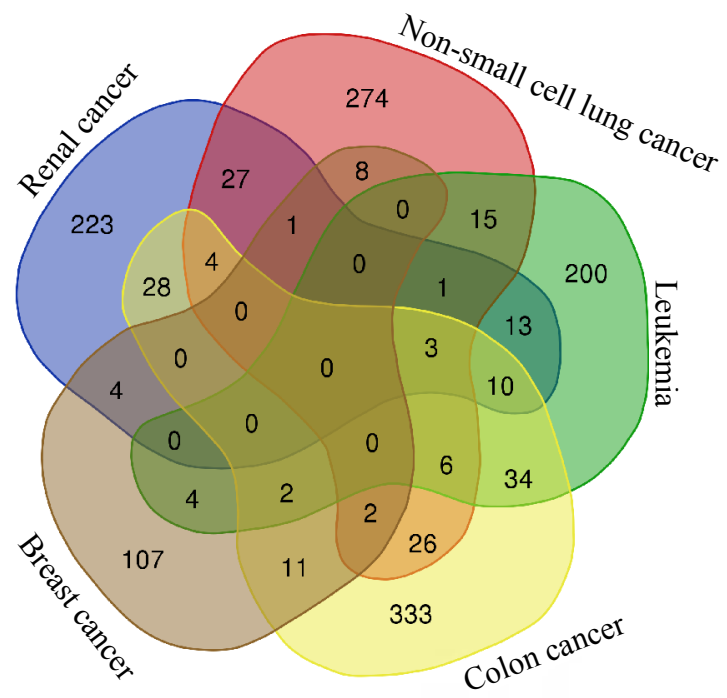

Figure 1. Number of identified differentially expressed genes (DEGs) in the presence of doxorubicin exposed to different cancer cell lines. with degree distribution following the power law model $\mathrm{P}(\mathrm{k}) \approx \mathrm{k}^{-0.856}$ with $\mathrm{R}^{2}=0.975$, (Supplementary Table S3 and Figure S1).

\subsubsection{Modular analysis of the active network}

The heavily connected clusters in the active network were determined in MCODE application, resulting in nine clusters (Supplementary Figure S2). The parameters for each cluster are summarized in Supplementary Table S4. Functional enrichment analyzes were performed for the identified clusters. Biological process terms/cellular compartments/molecular functions and pathways that were found to be statistically significant (BenjaminiHochberg p-value $<0.05)$ are presented for the first three clusters (Figures $3 \mathrm{~A}$ ).

Among the biological processes related to the genes/ proteins in Cluster 1, besides general terms such as transcription and translation, more specific processes such as ribosomal large subunit, ribosomal large subunit biogenesis, NIK/NF-kappaB signaling, tumor necrosis factor-mediated regulation of signaling pathway, and cell adhesion were also observed (Figure 3B). NIK/NFkappaB was reported to potentially trigger doxorubicin resistance (Gao et al., 2019). Ribosomes were frequently encountered in different terms in this cluster. This allows us to predict in advance that Cluster 1 is important in defining transcriptional regulatory networks.

In Cluster 2 (Figure 4A) there are no significant (Benjamini-Hochberg p-value $<0.05$ ) results for biological processes, but many for pathways (Figure 4B). Many cancer-related terms have been obtained, such as nonsmall cell lung cancer, glioma, viral carcinogenesis, melanoma, chronic myeloid leukemia, microRNAs in cancer, serotonergic synapse, thyroid hormone signaling pathway, thyroid cancer, bladder cancer, proteoglycans in cancer, regulation of actin cytoskeleton, endometrial cancer, acute myeloid leukemia, central carbon metabolism in cancer, renal cell carcinoma, prostate cancer, and choline metabolism in cancer. In addition, the genes in Cluster 2 were found to be associated with several signaling pathways that can be associated with cancer, long-term depression, and natural killer cell-mediated cytotoxicity.

Statistically significant biological processes associated with the genes/proteins that constitute Cluster 3 (Figure $5 \mathrm{~A}$ ) are cell cycle regulation, DNA damage checkpoint, and negative regulation of transcription from the RNA polymerase II promoter (Figure 5B). Significant molecular function terms are mostly related to binding. HTLV-I infection is the only significant pathway in Cluster 3.

\subsubsection{Transcriptional regulatory networks}

Identification of the transcription factors (TFs) responsible for the regulation of genes, which had altered expression is important to understand the working mechanism of doxorubicin. Transcription factor enrichment analysis for the three statistically significant clusters was performed in 
TAYMAZ-NIKKEREL / Turk J Biol
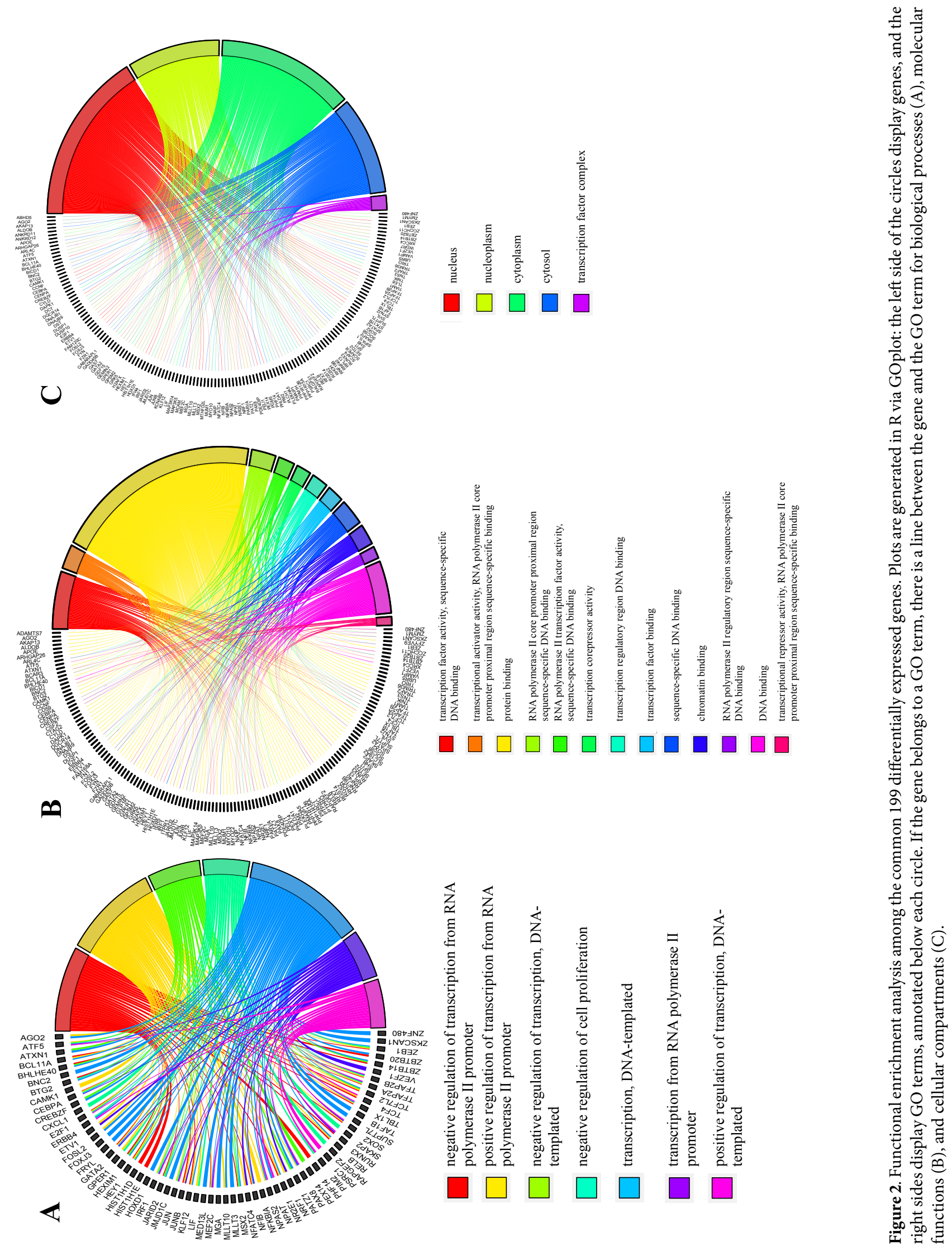


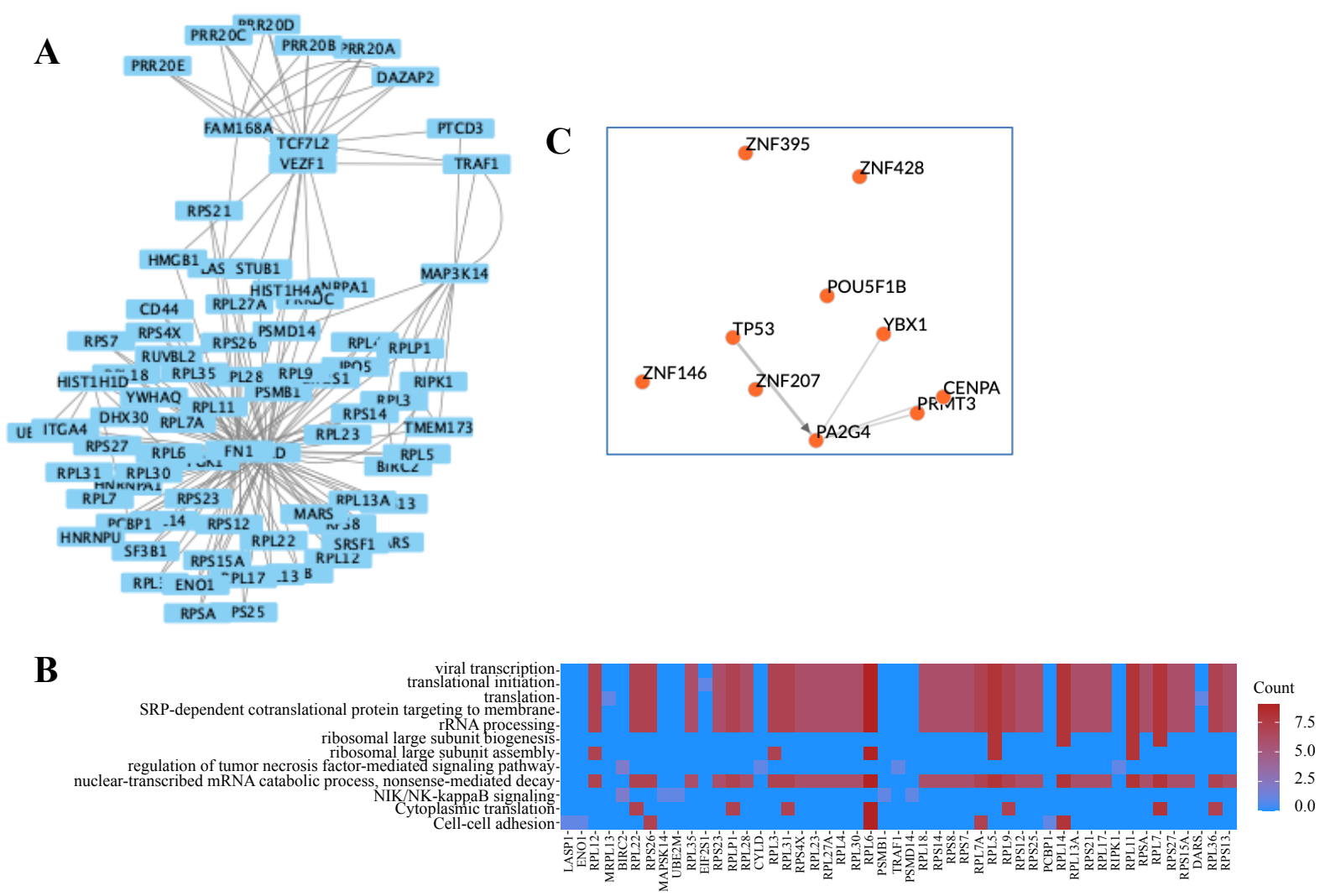

Figure 3. Modular representation of Cluster 1 (A), significant (Benjamini-corrected p-value < 0.05) biological process gene ontology (GO) terms in Cluster 1 (B), transcription factor enrichment for Cluster 1 (C).

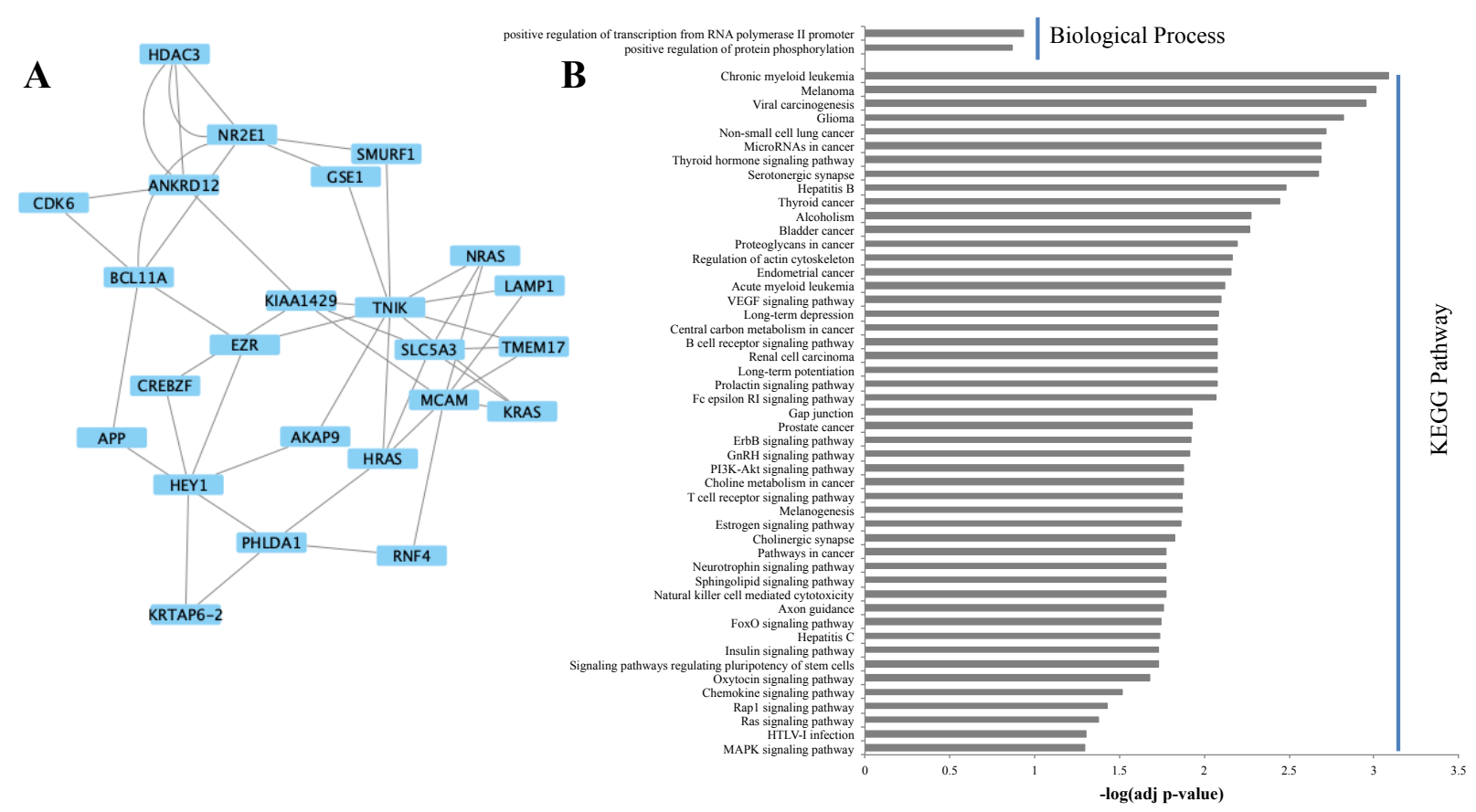

Figure 4. Modular representation of Cluster $2(\mathrm{~A})$, significant (Benjamini-corrected p-value $<0.05$ ) biological process gene ontology (GO) terms and KEGG pathways in Cluster 2 (B). 

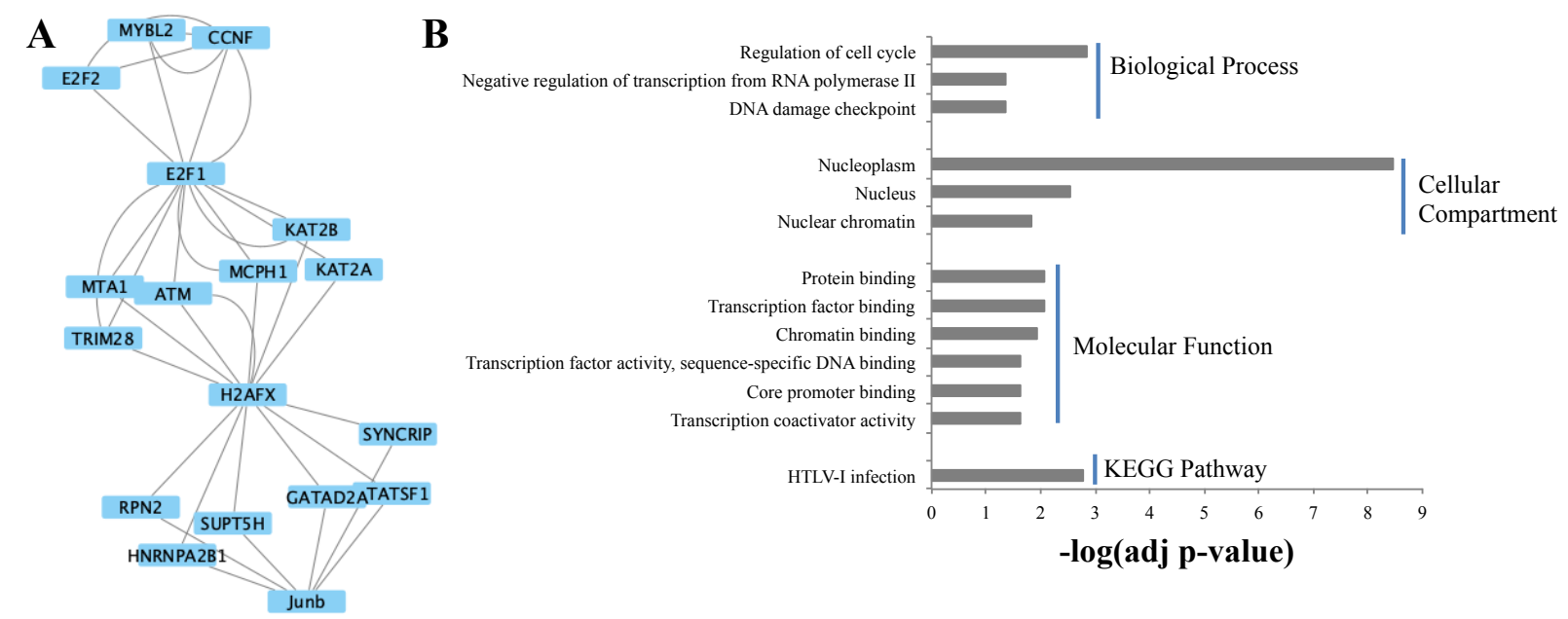

Figure 5. Modular representation of Cluster 3 (A), significant (Benjamini-corrected p-value $<0.05$ ) biological process, molecular function, cellular compartment gene ontology (GO) terms, and KEGG pathways in Cluster 3 (B).

ChEA3 (Keenan et al., 2019). TF co-expression networks help visualize top-ranked transcription factors in the framework of the larger human transcription regulation network. TF-TF co-regulatory networks are dynamically created using the best results of the selected library. The regulatory network for the top $10 \mathrm{TF}$ results for Cluster 1 is presented in Figure 3C.

Expression of TF Pou5flbp associated with Cluster 1 was shown to increase in cervical cytology and was proposed as a biomarker to identify cervical high-grade squamous lesions (Chen et al., 2020). Another TF, Znf428p, was presented as one of the regulators of cell invasion and cell migration in tumor cells (Barreiro-Alonso et al., 2018); it is also one of the seven panel biomarker candidates for the early diagnosis of hepatocellular carcinoma (Zhang et al., 2020). The transcription factor Prmt3p (protein arginine methyltransferase 3) has been shown to be dysregulated in gemcitabine-resistant pancreatic cancer cells. Overexpression of Prmt $3 p$ resulted in increased resistance to gemcitabine in pancreatic cancer cells, while reduction of Prmt3p was observed to restore gemcitabine sensitivity in resistant cells (Hsu et al., 2018). Based on this, inhibition of Prmt3p was proposed as a new strategy for the treatment of gemcitabine-resistant pancreatic cancer (Hsu et al., 2018).

The regulatory network for the top 10 TFs of Cluster 2 is presented in Supplementary Figure S3. Znf12p was proposed as a new target in imatinib-resistant gastrointestinal stromal tumor cells (Cao et al., 2018). Znf888p was reported as one of the methylation-based genes associated with clear-cell renal cell carcinoma (Wang et al., 2020). Overexpression of another Cluster 2-related TF, Ergp, was reported to be associated with tumor stage in prostate cancer but did not strongly predict the rate of relapse or death among men treated with radical prostatectomy (Pettersson et al., 2012). TMPRSS2ERG gene fusions are the major subtype of prostate cancer and are predominantly seen in young patients and lead to constitutive overexpression of the transcription factor Ergp. ERG overexpression alone is not prognostic; however, ERG was shown to modulate the expression of $>1600$ genes in prostate epithelial cells (Büscheck et al., 2019).

The regulatory network for the top 10 TFs of Cluster 3 is presented in Supplementary Figure S4. Of the TFs associated with Cluster 3, Tfdplp has the highest meanrank. Extensive characterization of DNA amplification at chromosome region 13q34 in breast cancer has revealed Tfdplp as one of the possible candidate target genes; in addition, tumors with high gene expression have been associated with markers of tumor proliferation and cell cycle progression (Melchor et al., 2009). Under normal physiological conditions and in most cancer cells, Tfdplp is a predominant protein that binds to E2F; deregulated TFDP1/E2F1 is known to induce stress leading to high levels of p53 in cancerous cells (Zhan et al., 2017).

Foxmlp associated with Cluster 3 is an oncogenic transcription factor that is overexpressed in most human cancers. Foxm $1 \mathrm{p}$ is involved in cell migration, invasion, angiogenesis, and metastasis. The important role of Foxm $1 p$ in cancer confirms its importance for therapeutic intervention (Halasi and Gartel, 2013). Understanding the regulation and function of Foxmlp has gained attention, which will provide potential roles of it in cancer and additional diseases.

Another TF associated with Cluster 3 is Cenpa (centromere protein-A), which was shown to be important in hepatocellular carcinoma development (Zhang et al., 
$2020)$, and gastric cancer progression and prognosis $(\mathrm{Xu}$ et al., 2020).

\section{Conclusion}

The analyses reported in this study have shown that doxorubicin causes common changes in gene expression in different types of cancer. Significant differential expressions were mainly observed in genes that have a role in transcription, regulation of transcription, and regulation of cell proliferation. Binding of protein, chromatin, and DNA are among significant molecular functions in addition to several terms related to RNA polymerase II activity, indicating that doxorubicin targets transcription machinery of RNA polymerase II, related to DNA intercalating property of doxorubicin. Further analysis of the PPI network revealed the interconnectivity of expression-level effects of doxorubicin on additional cancer types such as glioma, melanoma, thyroid, bladder, endometrial, and prostate cancers.

Identification of TFs within the significant clusters of the active network revealed the necessity of a comprehensive

\section{References}

Acharya N, Singh KP (2021). Differential sensitivity of renal carcinoma cells to doxorubicin and epigenetic therapeutics depends on the genetic background. Molecular and Cellular Biochemistry 476 (6): 2365-2379.

Ashrafizaveh S, Ashrafizadeh M, Zarrabi A, Husmandi K, Zabolian A et al. (2021). Long non-coding RNAs in the doxorubicin resistance of cancer cells. Cancer Letters 508: 104-114.

Barreiro-Alonso A, Lamas-Maceiras M, García-Díaz R, RodríguezBelmonte E, Yu L, et al. (2018). Delineating the HMGB1 and HMGB2 interactome in prostate and ovary epithelial cells and its relationship with cancer. Oncotarget 9 (27): 19050-19064.

Büscheck F, Zub M, Heumann A, Hube-Magg C, Simon R et al. (2019). The independent prognostic impact of the GATA2 pioneering factor is restricted to ERG-negative prostate cancer. Tumour Biology 41 (7): 1010428318824815.

Cao J, Wei J, Yang P, Zhang T, Chen Z et al. (2018). Genome-scale CRISPR-Cas 9 knockout screening in gastrointestinal stromal tumor with Imatinib resistance. Molecular Cancer 17 (1): 121.

Cava C, Sabetian S, Castiglioni I (2021). Patient-specific network for personalized breast cancer therapy with multi-omics data. Entropy (Basel) 23 (2).

Chen L, Dong B, Gao H, Xue H, Pan D et al. (2020). HPV-16 E2/E6 and POU5F1B as biomarkers to determine cervical high-grade squamous lesions and more. Journal of Inflammation Research 13: 813-821.

Di Francesco M, Celia C, Cristiano MC, d'Avanzo N, Ruozi B et al. (2021). Doxorubicin hydrochloride-loaded nonionic surfactant vesicles to treat metastatic and non-metastatic breast cancer. ACS Omega 6 (4): 2973-2989. understanding of the regulation principles of these TFs in order to provide new potential therapeutic strategies for cancer therapy. As a result of this study, Pou5f1b, Znf428, Prmt3, Znf12, Erg, Tfdp1, Foxm1, and Cenpa can be suggested as new drug targets in the development of drugs that can be effective in application to different cancer types.

The common effects of doxorubicin on human cancer cells were investigated at the transcriptome and interactome levels and evaluated with an integrated systems biology approach. In more detailed studies in the future, the information on intracellular metabolite levels, metabolic flux values and protein amounts may be integrated and thus the mechanisms affected will be detailed. In addition, the results obtained in this study showed the applicability of the proposed systems biology approach to other drugs/ drug types to obtain valuable information about the mechanism(s) of the drug in question. And in this way, new drug targets can be suggested as here.

\section{Acknowledgment and/or disclaimers}

This study has been supported by İstanbul Bilgi University Research Fund through Project No. 2019.01.009.

Ferreira A, Cunha-Oliveira T, Simões RF, Carvalho FS, Burgeiro A et al. (2017). Altered mitochondrial epigenetics associated with subchronic doxorubicin cardiotoxicity. Toxicology 390: 63-73.

Gao L, Han H, Wang H, Cao L, Feng WH (2019). IL-10 knockdown with siRNA enhances the efficacy of doxorubicin chemotherapy in EBV-positive tumors by inducing lytic cycle via PI3K/p38 MAPK/NF-kB pathway. Cancer Letters 462: 12-22.

Gopi LK, Kidder BL (2021). Integrative pan cancer analysis reveals epigenomic variation in cancer type and cell specific chromatin domains. Nature Communications 12 (1): 1419.

Halasi M, Gartel AL (2013). Targeting FOXM1 in cancer. Biochemical Pharmacology 85 (5): 644-652.

Hsu MC, Pan MR, Chu PY, Tsai YL, Tsai CH et al. (2018). Protein arginine methyltransferase 3 enhances chemoresistance in pancreatic cancer by methylating hnRNPA1 to increase ABCG2 expression. Cancers (Basel) 11 (1).

Huang D, Sherman B, Lempicki R (2009). Bioinformatics enrichment tools: paths toward the comprehensive functional analysis of large gene lists. Nucleic Acids Research 37 (1): 1-13.

Karabekmez ME, Taymaz-Nikerel H, Eraslan S, Kirdar B (2021). Time-dependent re-organization of biological processes by the analysis of the dynamic transcriptional response of yeast cells to doxorubicin. Molecular Omics 17 (4): 572-582.

Keenan AB, Torre D, Lachmann A, Leong AK, Wojciechowicz ML et al. (2019). ChEA3: transcription factor enrichment analysis by orthogonal omics integration. Nucleic Acids Research 47 (W1): W212-W224. 
Maillet A, Tan K, Chai X, Sadananda SN, Mehta A et al. (2016). Modeling doxorubicin-induced cardiotoxicity in human pluripotent stem cell derived-cardiomyocytes. Scientific Reports 6: 25333.

Melchor L, Saucedo-Cuevas LP, Muñoz-Repeto I, Rodríguez-Pinilla SM, Honrado E et al. (2009). Comprehensive characterization of the DNA amplification at 13q34 in human breast cancer reveals TFDP1 and CUL4A as likely candidate target genes. Breast Cancer Research 11 (6): R86.

Monks A, Zhao Y, Hose C, Hamed H, Krushkal J et al. (2018). The NCI transcriptional pharmacodynamics workbench: a tool to examine dynamic expression profiling of therapeutic response in the NCI-60 cell line panel. Cancer Research 78 (24): 68076817.

Oughtred R, Stark C, Breitkreutz BJ, Rust J, Boucher L et al. (2019). The BioGRID interaction database: 2019 update. Nucleic Acids Research 47 (D1): D529-D541.

Pettersson A, Graff RE, Bauer SR, Pitt MJ, Lis RT et al. (2012). The TMPRSS2: ERG rearrangement, ERG expression, and prostate cancer outcomes: a cohort study and meta-analysis. Cancer Epidemiology Biomarkers \& Prevention 21 (9): 1497-1509.

Selevsek N, Caiment F, Nudischer R, Gmuender H, Agarkova I et al. (2020). Network integration and modelling of dynamic drug responses at multi-omics levels. Communications Biology 3 (1): 573 .

Shahriari M, Taghdisi SM, Abnous K, Ramezani M, Alibolandi M (2021). Self-targeted polymersomal co-formulation of doxorubicin, camptothecin and FOXM1 aptamer for efficient treatment of non-small cell lung cancer. Journal of Controlled Release 335: 369-388.

Shannon P, Markiel A, Ozier O, Baliga N, Wang J et al. (2003). Cytoscape: A software environment for integrated models of biomolecular interaction networks. Genome Research 13 (11): 2498-2504.

Sonowal H, Pal PB, Wen JJ, Awasthi S, Ramana KV et al. (2017). Aldose reductase inhibitor increases doxorubicin-sensitivity of colon cancer cells and decreases cardiotoxicity. Scientific Reports 7 (1): 3182.
Taymaz-Nikerel H, Karabekmez ME, Eraslan S, Kirdar B (2018). Doxorubicin induces an extensive transcriptional and metabolic rewiring in yeast cells. Scientific Reports 8 (1): 13672.

Turk S, Turk C, Akbar MW, Kucukkaraduman B, Isbilen M et al. (2020). Renin angiotensin system genes are biomarkers for personalized treatment of acute myeloid leukemia with doxorubicin as well as etoposide. PLoS One 15 (11): e0242497.

van der Zanden SY, Qiao X, Neefjes J (2021). New insights into the activities and toxicities of the old anticancer drug doxorubicin. FEBS Journal 288 (21):6095-6111.

Wang J, Zhang Q, Zhu Q, Liu C, Nan X et al. (2020). Identification of methylation-driven genes related to prognosis in clear-cell renal cell carcinoma. Journal of Cellular Physiology 235 (2): 1296-1308.

Xu Y, Liang C, Cai X, Zhang M, Yu W et al. (2020). High centromere protein-A (CENP-A) expression correlates with progression and prognosis in gastric cancer. OncoTargets and Therapy 13: 13237-13246.

Yang F, Teves SS, Kemp CJ, Henikoff S (2014). Doxorubicin, DNA torsion, and chromatin dynamics. Biochimica et Biophysica Acta 1845 (1): 84-89.

Yu K, Chen B, Aran D, Charalel J, Yau C et al. (2019). Comprehensive transcriptomic analysis of cell lines as models of primary tumors across 22 tumor types. Nature Communications 10 (1): 3574 .

Zhan W, Wang W, Han T, Xie C, Zhang T et al. (2017). COMMD9 promotes TFDP1/E2F1 transcriptional activity via interaction with TFDP1 in non-small cell lung cancer. Cellular Signalling 30: 59-66.

Zhang S, Liu Y, Chen J, Shu H, Shen S et al. (2020). Autoantibody signature in hepatocellular carcinoma using seromics. Journal of Hematology \& Oncology 13 (1): 85.

Zhang Y, Yang L, Shi J, Lu Y, Chen X et al. (2020). The oncogenic role of CENPA in hepatocellular carcinoma development: evidence from bioinformatic analysis." BioMed Research International 2020: 3040839 . 
Supplementary Files for

Doxorubicin-induced transcriptome meets interactome: identification of new drug targets

Table S1: Doxorubicin-induced transcriptome data used in the study: cancer type, cell line, GEO accession numbers of the control and case gene expression data

\begin{tabular}{|c|c|c|c|}
\hline Cancer type & Cell Line & GEO control & GEO case \\
\hline Renal cancer & $786-0$ & $\begin{array}{l}\text { GSM3233605 } \\
\text { GSM3233606 } \\
\text { GSM3233607 }\end{array}$ & $\begin{array}{l}\text { GSM3233608 } \\
\text { GSM3233609 } \\
\text { GSM3233610 }\end{array}$ \\
\hline Breast cancer & MCF7 & $\begin{array}{l}\text { GSM3233810 } \\
\text { GSM3233811 } \\
\text { GSM3233812 }\end{array}$ & $\begin{array}{l}\text { GSM3233815* } \\
\text { GSM3233816 } \\
\text { GSM3233817 }\end{array}$ \\
\hline Leukemia & CCRF-CEM & $\begin{array}{l}\text { GSM3233659 } \\
\text { GSM3233660 } \\
\text { GSM3233661 }\end{array}$ & $\begin{array}{l}\text { GSM3233662 } \\
\text { GSM3233663 } \\
\text { GSM3233664 }\end{array}$ \\
\hline Non-small cell lung cancer & A549/ATCC & $\begin{array}{l}\text { GSM3233623 } \\
\text { GSM3233624 } \\
\text { GSM3233625 }\end{array}$ & $\begin{array}{l}\text { GSM3233626 } \\
\text { GSM3233627 } \\
\text { GSM3233628 }\end{array}$ \\
\hline Colon cancer & COLO 205 & $\begin{array}{l}\text { GSM3233668 } \\
\text { GSM3233669 } \\
\text { GSM3233670 }\end{array}$ & $\begin{array}{l}\text { GSM3233671 } \\
\text { GSM3233672 } \\
\text { GSM3233673 }\end{array}$ \\
\hline
\end{tabular}

* $100 \mathrm{~nm}$ of doxorubicin was administered.

Table S2: Differentially expressed common genes under doxorubicin treatment in different cancer types

\begin{tabular}{|c|c|c|c|c|c|c|c|}
\hline ABCA2 & CENPA & FOSL2 & IGHG1 & MED13L & PEX14 & RPL13 & TIAM1 \\
\hline ABHD5 & COL6A1 & FOXJ3 & IRF1 & MEF2C & PGM5 & RUNX3 & TLE6 \\
\hline ADAMTS7 & CREBZF & FRYL & ITPR1 & MGA & PHF14 & SCAF4 & TNFRSF10D \\
\hline $\mathrm{AGO} 2$ & CSF2 & FYN & JARID2 & MLLT10 & PHLDA1 & SCAPER & TNFSF9 \\
\hline AKAP13 & CSHL1 & FZR1 & JMJD1C & MLLT3 & PIM2 & SETD2 & TNIK \\
\hline ALDOB & CTAG2 & GABARAPL1 & JUN & MSX2 & PLCH1 & SEZ6L & TNS3 \\
\hline ANKRD11 & CXCL1 & GADD45B & JUNB & MTHFD2L & PLEKHA5 & SFI1 & TRAF2 \\
\hline ANKRD12 & CXCL3 & GAS7 & KCNAB2 & MUM1 & PMAIP1 & SHB & TRIM36 \\
\hline APOE & CYLD & GATA2 & KLF12 & MYO10 & PPM1H & SIPA1L1 & TRIO \\
\hline ARHGAP26 & DAPK1 & GDF15 & LARGE & MYOZ2 & PPP1R12B & SKAP2 & TRPM3 \\
\hline ARL4C & DCT & GEMIN2 & LCT & NAIP & PPP1R15A & SLC19A2 & TSPAN5 \\
\hline ARMC9 & DGCR14 & GLP1R & LIF & NFATC4 & PSD3 & SLC5A3 & UBR5 \\
\hline ATF5 & DNAJB1 & GPER1 & LILRA5 & NFIB & PSRC1 & socs6 & VAMP1 \\
\hline ATXN1 & DNAJB9 & GPR39 & LIMCH1 & NFKBIA & PTPRC & SOX2 & VEZF1 \\
\hline BCAR3 & DST & GPSM2 & LINS1 & NPAS2 & PTPRO & SPATA2 & WDR7 \\
\hline BCL11A & DUSP1 & GRK5 & LOC 100287590 & NPAT & RAB31 & STK17B & XRCC4 \\
\hline BHLHE40 & DUSP10 & H2AFX & RUNX1 & NR2E1 & RALGPS1 & SUPT7L & ZBTB14 \\
\hline BICD1 & E2F1 & HEXIM1 & WNK1 & NRF1 & RAPGEF2 & SVIL & ZBTB20 \\
\hline $\mathrm{BNC} 2$ & ERBB4 & HEY1 & LOC643733 & OGFOD2 & RELB & SYNJ2 & $\mathrm{ZCCHC11}$ \\
\hline BTG2 & ETV1 & HIST1H1D & LPCAT4 & PAQR6 & RFX7 & TAF1B & ZEB1 \\
\hline C16orf71 & FAM120C & HIST1H1E & LRIG1 & PARVA & RGS14 & TBL1X & ZFYVE9 \\
\hline CAMK1 & FAM168A & HLA-E & LTB4R & PATZ1 & RGS2 & TCF4 & ZKSCAN1 \\
\hline CCNF & FAM193A & HOXD1 & MAP3K14 & PAX8 & $\mathrm{RHOH}$ & TCF7L2 & ZMYM1 \\
\hline CD59 & FGF18 & IER5 & MAP3K5 & PDE4DIP & RNF19B & TFAP2A & ZNF480 \\
\hline CEBPA & FN1 & IFI44 & MCAM & PELI1 & RNF40 & TFAP2B & \\
\hline
\end{tabular}




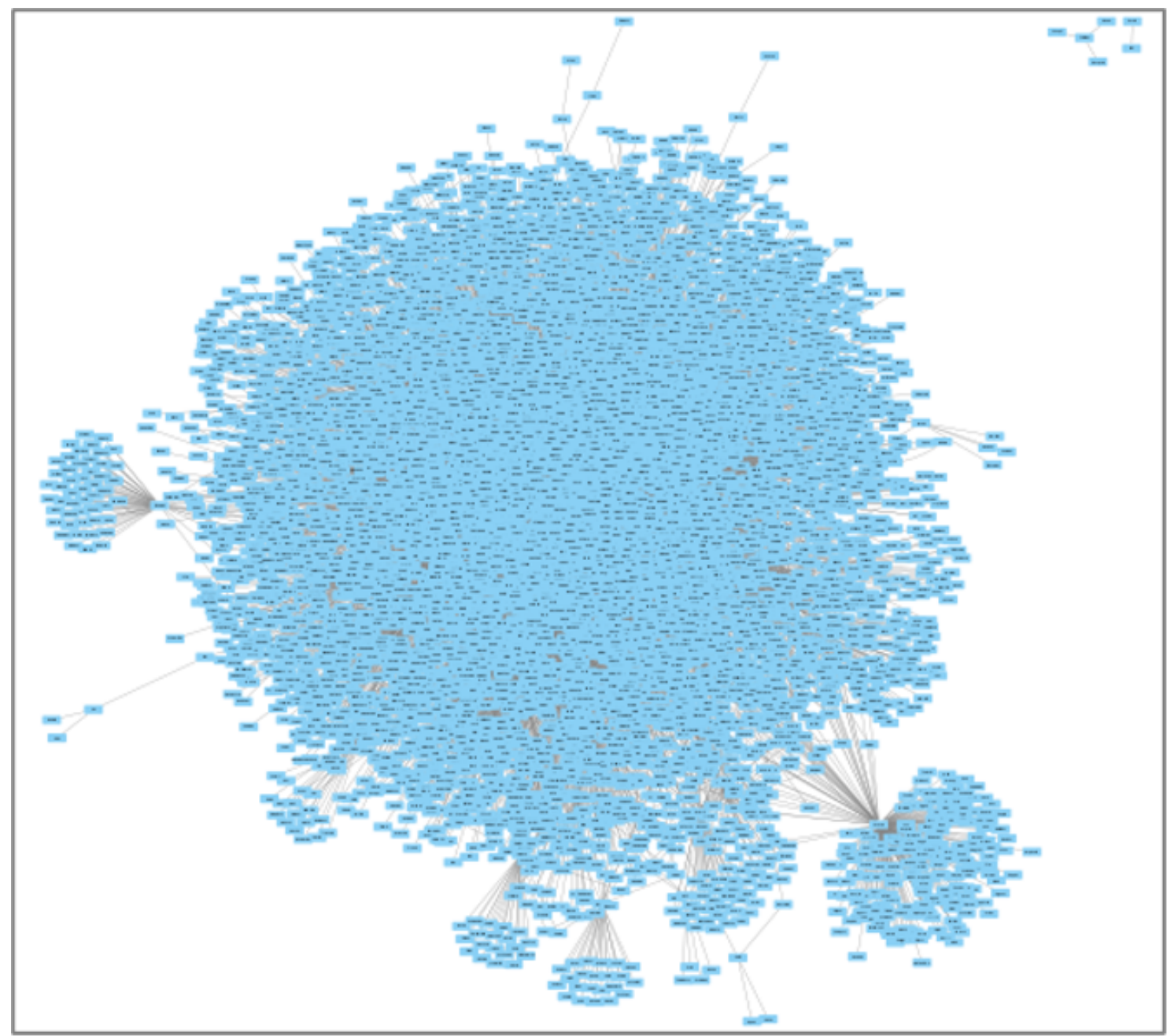

Figure S1: Active network constructed by protein-protein interactions of proteins encoded by the genes that are commonly differentially expressed under doxorubicin treatment in different cancer types

Table S3: Parameters of the active PPI (protein-protein interaction) network

\begin{tabular}{|l|l|l|l|}
\hline Clustering coefficient : & 0.045 & Number of nodes : & 7022 \\
\hline Connected components : & 3 & Network density : & 0.001 \\
\hline Network diameter : & 9 & Network heterogeneity : & 5.726 \\
\hline Network radius : & 1 & ISolated nodes : & 0 \\
\hline Network centralization : & 0.122 & Number of self-loops : & 0 \\
\hline Shortest paths : & $49217254(99 \%)$ & Multi-edge node pairs : & 1309 \\
\hline Characteristic path length : & 3.845 & & \\
\hline Avg. number of neighbours : & 4.120 & & \\
\hline
\end{tabular}


TAYMAZ-NIKKEREL / Turk J Biol

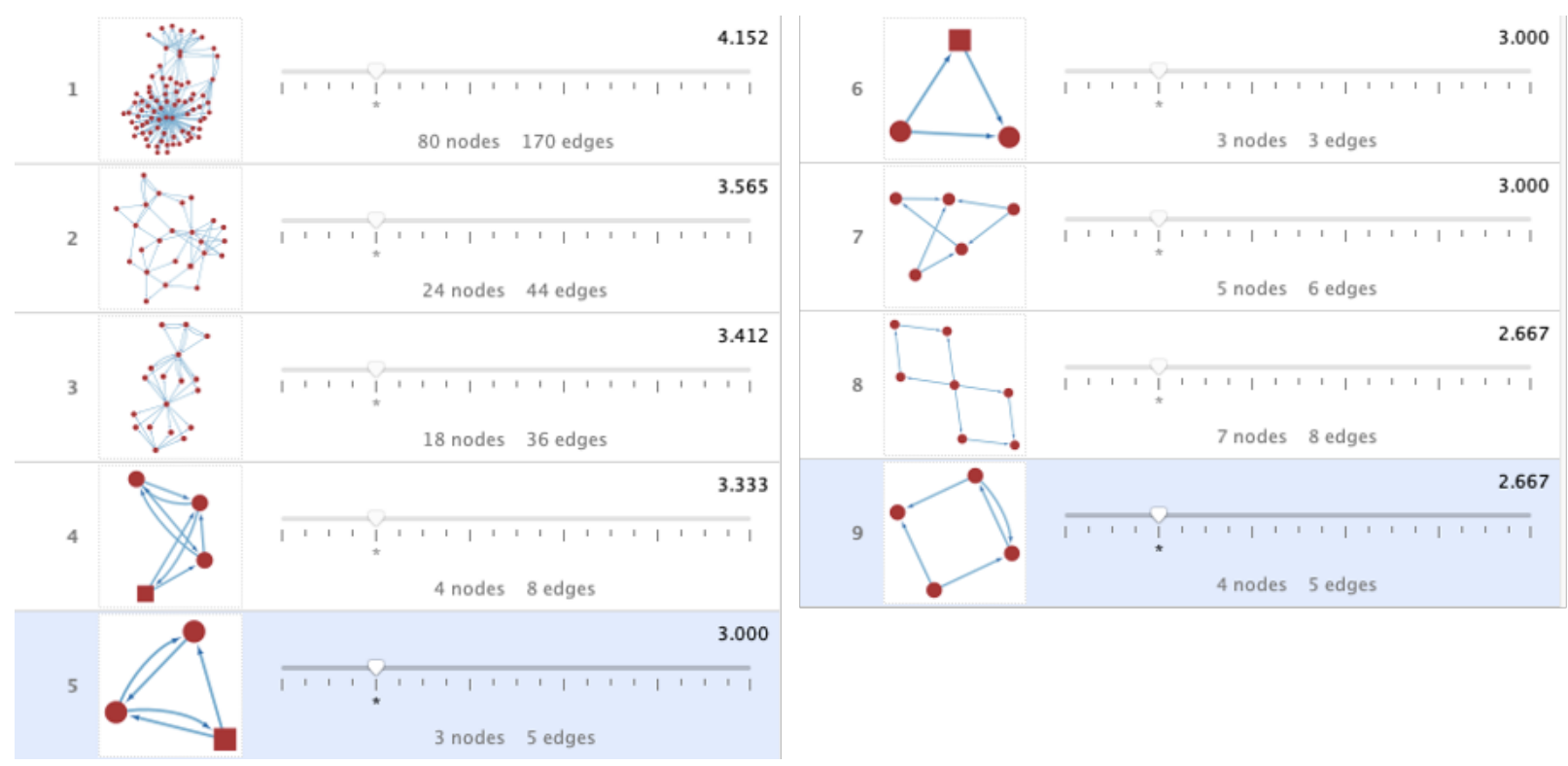

Figure S2: Clusters within the active PPI (protein-protein interaction) network. Clusters were identified via MCODE application in Cytoscape.

Table S4: Parameters of the clusters within the active PPI (protein-protein interaction) network

\begin{tabular}{|l|l|l|l|}
\hline Cluster & $\begin{array}{l}\text { Score } \\
\left(\begin{array}{l}\text { Density } \\
\text { of edges) }\end{array}\right.\end{array}$ & $\begin{array}{l}\text { Number of } \\
\text { nodes }\end{array}$ & $\begin{array}{l}\text { Number of } \\
\text { edges }\end{array}$ \\
\hline 1 & 4.152 & 80 & 170 \\
\hline 2 & 3.565 & 24 & 44 \\
\hline 3 & 3.412 & 18 & 36 \\
\hline 4 & 3.333 & 4 & 8 \\
\hline 5 & 3 & 3 & 5 \\
\hline 6 & 3 & 3 & 3 \\
\hline 7 & 3 & 5 & 6 \\
\hline 8 & 2.667 & 7 & 8 \\
\hline 9 & 2.667 & 4 & 5 \\
\hline
\end{tabular}



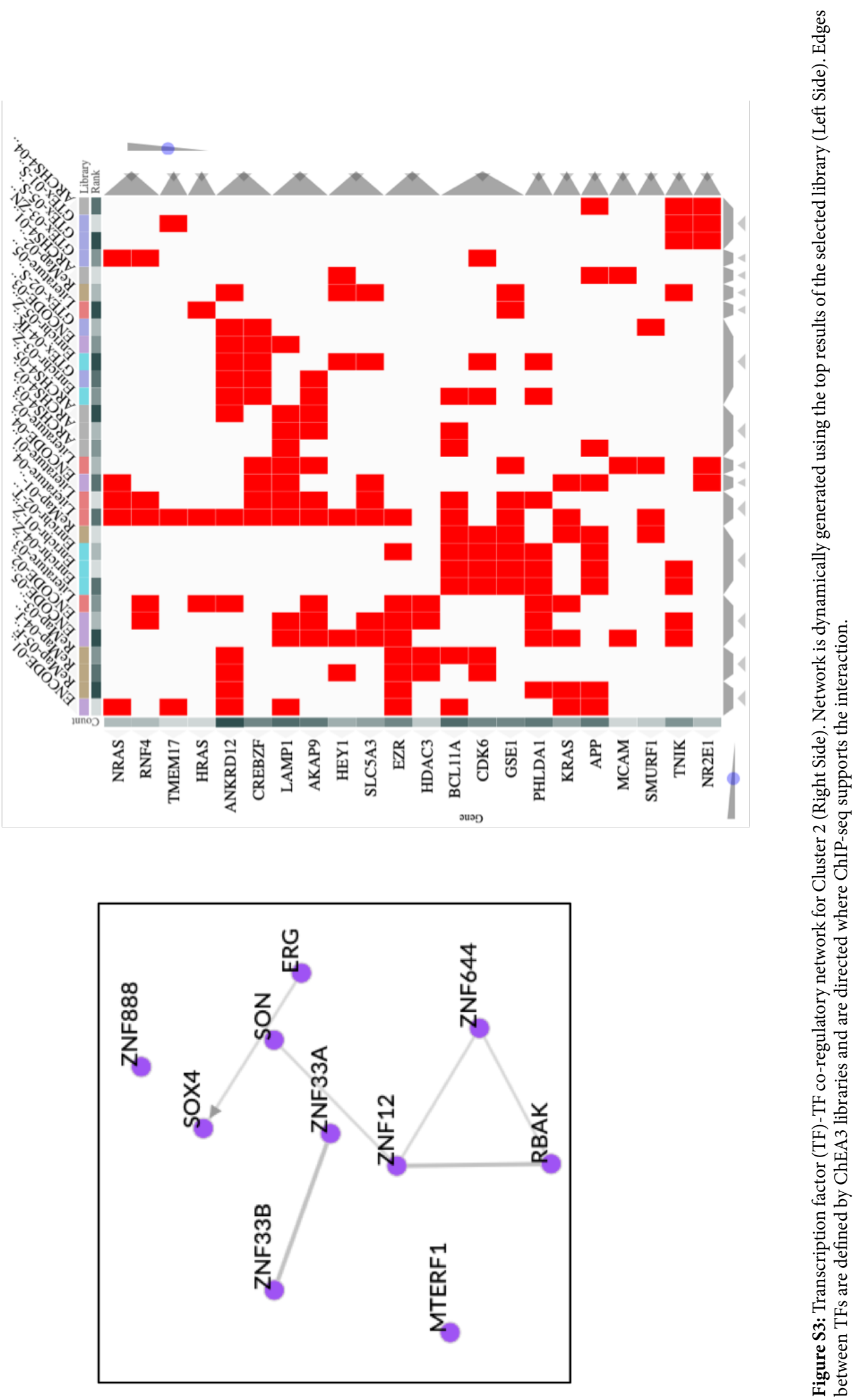

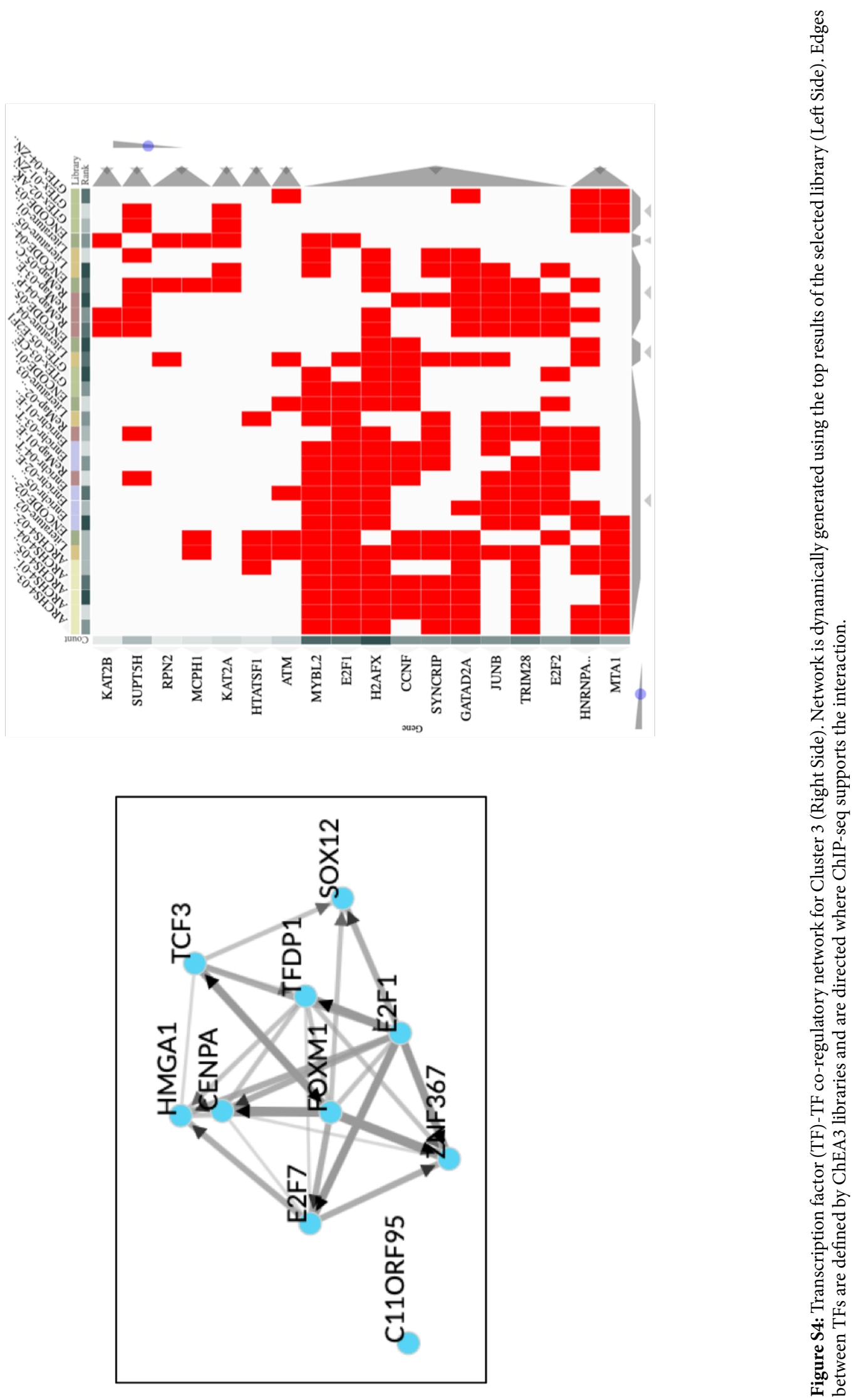